\title{
Species-specific effect of UV-B radiation on the temporal pattern of leaf growth
}

\author{
Thomas Matthew Robson* and Pedro José Aphalo \\ Department of Biosciences, Plant Biology, University of Helsinki, P.O. Box 65, Helsinki 00014 Finland
}

\author{
Correspondence \\ *Corresponding author, \\ e-mail: matthew.robson@helsinki.fi \\ Received 8 April 2011; \\ revised 27 August 2011 \\ doi:10.1111/j.1399-3054.2011.01546.x
}

\begin{abstract}
Recent molecular and physiological studies have demonstrated that ultraviolet-B radiation (UV-B) can affect some of the processes involved in leaf growth, but the phases of leaf growth affected have not been clearly delimited. We used functional growth analysis to assess the effects of UV-B radiation on the time course of leaf growth in seedlings of two birch species (Betula pendula and Betula pubescens). Our aim was to identify the phase(s) of leaf development affected by UV-B radiation. In a greenhouse study, 1-year-old birch seedlings were subjected to three daily doses of supplemental UV-B radiation treatments $\left(U V-B^{+}\right)$and no UV-B radiation controls $\left(U V-B^{-}\right)$. Leaf growth measurements every 2 days were complemented by assessment of other functional traits over a 4-week period at the start of the growing season. Using fitted curves, we were able to determine that the rate of leaf expansion was slowed by the UV- $\mathrm{B}^{+}$treatment in leaves of $B$. pendula because of a slower maximum leaf growth rate compared with plants under the UV-B ${ }^{-}$controls, but that compensation toward the end of the period of expansion negated this difference when leaves reached their final size. UV-B ${ }^{+}$had little effect on the rate of $B$. pubescens leaf growth despite a larger reduction in leaf final size due to $U V-B^{+}$than occurred in $B$. pendula leaves. In conclusion, effective regulation ameliorated the effects of UV-B radiation on leaf and seedling growth in $B$. pendula, whereas in $B$. pubescens, reductions in leaf final size under $U \mathrm{~V}-\mathrm{B}^{+}$were consistent with a slightly reduced rate of height growth.
\end{abstract}

\section{Introduction}

Research into the influence of solar ultraviolet-B (UV-B) radiation on plants is moving beyond assessments of the modest detrimental effects of increased solar UV$\mathrm{B}$ radiation to consider the possible regulatory role of ambient UV-B radiation doses (Ballaré et al. 2010). A mechanistic approach has revealed UV-B-induced gene expression producing photomorphogenic responses in the model species Arabidopsis thaliana (Jenkins 2009). However, the consequences of these UV-B radiation effects for plant performance and their consistency across other species remain unclear. Betula pendula and Betula pubescens are two species whose responses to UV-B radiation have been widely studied because of their prominence at high northern latitudes where ozone depletion has altered natural solar UV-B radiation doses (de la Rosa et al. 2003, Tegelberg et al. 2004, JulkunenTiitto et al. 2005, Kotilainen et al. 2009, Morales et al. 2010). As such, they are good subjects for investigation to develop our understanding of the effects of UV-B radiation from the mechanistic and physiological levels up to the scale of plant growth responses.

Abbreviations - RCD, root collar diameter; UV-A, ultraviolet-A radiation of wavelength 315-400 nm; UV-B, ultraviolet-B radiation of wavelength $280-315 \mathrm{~nm}$; UV-B ${ }^{+}$, supplemental UV-B radiation treatments; UV-B- , supplemental UV-B radiation control treatments with filters blocking all the UV-B radiation but transmitting the small amount of UV-A radiation from the lamps. 
In ecology, the effect of environmental stress is usually considered by monitoring responses of the whole plant or its organs; however, this approach fails to make a mechanistic link between the stress applied and the reaction recorded. While a bottom-up approach has the drawback that the relevance of effects at physiological and morphological levels can be difficult to interpret ecologically because often these effects do not scale up to the whole plant level. Growth analysis bridges the gap between growth of the whole plant and the fate of its cells, by detailing the growth of component organs and tissues (Hunt 2003).

Recent attempts to scale from the mechanistic effects of UV-B radiation on cells to the leaf scale have had mixed success. Leaf size and leaf expansion rate are only inconsistently decreased by UV-B radiation, and results are often specific to particular species and studies. For instance, Hectors et al. (2010) reported reduced leaf size in $A$. thaliana plants under UV radiation was attributable only to reduced cell expansion (not cell number), which also produced a transient reduction in leaf length:width ratio during development (Hectors et al. 2010), whereas Wargent et al. (2009a) reported reduced cell division in $A$. thaliana plants receiving high doses of UV-B radiation. This effect was compensated by larger cell size in wild-type plants expressing the UV RESISTANCE LOCUS8 (UVR8), possibly associated with endoreduplication. This result contrasts with a reduced leaf expansion rate and final size in lettuce, which was linked to increased cell-wall peroxidase activity as a result of UV radiation under controlled conditions (Wargent et al. 2009b). However, in an equivalent field experiment at a dose of $9 \mathrm{~kJ} \mathrm{~m}^{-2}$ day $^{-1}$, ultravioletA (UV-A) radiation of wavelength $315-400 \mathrm{~nm}$ and UV-B radiation together significantly reduced upperepidermal cell size compared with zero UV radiation treatments, with roughly half of the reduction attributable to each waveband; although no consequential effects were detected on leaf biophysical properties resulting from this change (Wargent et al. 2009b), which might have reflected changes in cell-wall properties. Other biochemicals such as expansins, phenolics and auxins, all known to respond to $U V$, have also been suggested as the mediators of reduced cell expansion (Hectors et al. 2007, Brown and Jenkins 2008, Favory et al. 2009).

Following initial development from primordia, leaf growth declines from an exponential rate, with reduced cell division followed by slower expansion until a final size is attained (Tardieu et al. 1999, Granier and Tardieu 2009). We used a function formulated by Prunty (1983), that is piece-wise linear in the limit, to analyze leaf expansion data and test (hypothesis 1 ) whether seedlings of $B$. pubescens and B. pendula respond similarly to a range of UV-B radiation treatments compared with their respective control treatments lacking UV-B radiation. Growth analysis allowed us to distinguish (hypothesis 2) which of; leaf expansion rate; final leaf size and rate of leaf production, were specifically affected by the UV$B$ radiation treatments, and whether these differences increased in response to an increasing UV-B dose or were simply induced by UV-B radiation irrespective of dose. Finally, we assessed (hypothesis 3) whether the responses agreed with our existing understanding of the mechanisms of UV-B action on leaf growth gleaned from recent molecular studies, and (hypothesis 4) whether they matched our expectations given the ecological and physiological responses of birch species to UV$B$ radiation measured in this and past experiments. In each case, by applying three treatments of supplemental UV-B $\left(U V-B^{+}\right)$and their requisite controls, we could also examine whether the regulatory role of $U V$-B radiation at low doses contributed more to the treatment differences than any detrimental effect of UV-B radiation at high doses for these species.

\section{Materials and methods}

\section{Birch experiment}

One-year-old containerized seedlings of $B$. pendula and $B$. pubescens were moved from cold storage to a greenhouse during May when buds were still dormant. The seedlings had previously been reared at the Suonenjoki Research Station (Finnish Forest Research Institute) from provenanced seed of central Finnish origin.

From the outset, plants were exposed to a natural photoperiod of about $18 \mathrm{~h}$, and UV-B irradiation was administered centered on solar midday, to mimic the natural daily peak of UV-B exposure. Conditions in the greenhouse were controlled to maintain the temperature close to $20^{\circ} \mathrm{C}$ during the day and $15^{\circ} \mathrm{C}$ at night, and about $50 \%$ humidity (Thermohygrograph, Lambrecht 252, Goettingen, Germany).

Seedlings were planted in $2-1$ pots in a $45 \%$-peat to $55 \%$-grit substrate. Non-limiting water and nutrient conditions were maintained using a balanced fertilizer (Superex-6, Kekkilä, Finland) $100 \mathrm{ml}$ at $0.2 \mathrm{~g} \mathrm{I}^{-1}$ (equivalent to $0.02 \mathrm{~g}$ seedling $^{-1}$ day $^{-1}$ ).

Six treatments were administered to each species in a factorial design: two UV-B filter types, and three irradiation-time periods. The experimental units were arranged in three blocks in the greenhouse. Each experimental unit contained seven seedlings (sub-samples). The position of plants under each lamp-frame was rotated regularly, and a border of birch seedlings not included in the analysis encircled the treatments to eliminate potential edge effects under the filters. Cellulose 
diacetate filters $(0.115 \mathrm{~mm}$, FilmSales, London, UK) transparent to UV-B radiation were used for the treatments $\left(U V-B^{+}\right)$, and polyester filters $(0.125 \mathrm{~mm}$, FilmSales) provided UV-B-blocking UV-A radiation controls $\left(U V-B^{-}\right)$: i.e. control treatments produced by filters blocking all the UV-B radiation but transmitting the small amount of UV-A radiation emitted by lamps at each UV$\mathrm{B}^{+}$dose. The filters were positioned horizontally under the lamps, and so also filtered ambient light. The cellulose diacetate filters were replaced after $50 \mathrm{~h}$ of lamp use to avoid problems of deterioration. Vertical curtains of polyester film separated all the treatments. The UV-B lamps (Q-panel UV-B-313, 40 W, special 120-cm long fluorescent tubes, Cleveland, $\mathrm{OH}$ ) were maintained at $40 \mathrm{~cm}$ above the top of seedlings for the duration of the experiment by periodically raising the height of the lampframes. The UV dose from each lamp was calculated from measurements with a spectroradiometer (Macam SR9910-PC, Macam Photometrics, Livingstone, UK).

Biologically effective UV-B radiation was determined using Green's formulation of the generalized plantdamage spectral-weighting function normalized at 300 nm, 'GREEN' (Caldwell 1971, Green et al. 1974, Aphalo etal. 1999) and the plant growth action spectrum, 'PG' (Flint and Caldwell 2003). Plants received either (1) approximately $2.6 \mathrm{~kJ} \mathrm{~m}^{-2} \mathrm{day}^{-1}$ of UV-B GREEN radiation with lamps switched on for 60 min day $^{-1}$; a value similar to the growing season average for central Finland (Kotilainen et al. 2008): or (2) approximately $5.2 \mathrm{~kJ} \mathrm{~m}^{-2}$ day $^{-1}$ of UV-B GREEN radiation with lamps switched on for 120 min day $^{-1}$; slightly higher than a clear midsummer day in central Finland (Kotilainen et al. 2008): or (3) approximately $13.0 \mathrm{~kJ} \mathrm{~m}^{-2}$ day $^{-1}$ of UV$B_{\text {GREEN }}$ radiation with lamps switched on for $300 \mathrm{~min}$ $\mathrm{d}^{-1}$. The ratio of total effective irradiance from the cellulose diacetate-filtered lamps alone calculated with 'PG' compared to 'GREEN' was 0.95, with a small $(<10 \%)$ contribution by UV-A in the first case and none in the second case. For unweighted irradiances, the contribution of UV-A to total UV from the filtered lamps was $63 \%$, however, the amount of UV-A radiation emitted by the UV-B lamps is very low compared to the UV-A irradiance in sunlight. The photosynthetically active radiation in the greenhouse was measured at a photosynthetic photon flux density of $14 \mathrm{~mol} \mathrm{~m}^{-2}$ day $^{-1}$.

\section{Measurements}

The length and width of the first, third and fifth distal leaves on the first lateral branch of each seedling were measured to the nearest millimeter using calipers. This procedure was carried out on all the plants [three leaves $x$ seven plants $x$ three blocks $\times$ treatment combination (two UV-B ${ }^{+/-} \times$three UV doses $\times$two species) $=$ 756 leaves in total] at the same time of day, every second day, from bud burst until leaves were considered fully expanded. In some birch species, the size of leaves produced decreases as the growing season precedes but in $B$. pubescens a constant maximum leaf size is achieved, and because leaves on the main stem and lateral shoots behave differently (Kozlowski and Clausen 1966), consistency in the order and position of leaves measured is very important.

Leaves of all sizes from the border-birch seedlings (excluded from the experiment) were measured and harvested to provide a calibration of length, width and leaf area. These leaves were scanned (desk scan, HP) and leaf area was calculated using WINFOLIA 6.2 (Regent Instruments, Quebec, Canada). Linear regression of length $\times$ width measurements against the measured leaf area was carried out. All length and width measurements $(\mathrm{mm})$ were thus converted to leaf area $\left(\mathrm{mm}^{2}\right)[\mathrm{y}=(\mathrm{L} \times \mathrm{W}$ - A)/B] (B. pendula $A=0.60, B=1.635 R^{2}=0.996$; $B$. pubescens $A=0.48, B=1.477, R^{2}=0.995$ ) (Kvêt and Marshall 1971).

The size of each leaf was plotted and fitted curves were calculated for each leaf individually using the described function (Eqn 1). The rate of leaf production, and number of leaves produced were estimated. The ratio of leaf length to width was also calculated from these data to test for a change in leaf shape occurring over the course of the experiment and between treatments (Fig. 5; Supporting Information, Appendix S2, Fig. S4).

Height and root collar diameter (RCD) of seedlings were measured every 4 and 12 days, respectively (Appendix S2, Figs S5 and S6). Leaves were harvested after 29 days receiving the UV-B radiation treatments and immediately chilled to $3{ }^{\circ} \mathrm{C}$. Chlorophyll extraction was made on the same day as harvesting, two leaf disks per leaf were placed in $3 \mathrm{ml} \mathrm{N}, \mathrm{N}$-dimethylformide in the dark and shaken continuously at $3{ }^{\circ} \mathrm{C}$ for 5 days. Absorbance at 663.8 and $646.8 \mathrm{~nm}$ was measured using a spectrophotometer (HP 8453 UV-visible diode-array spectrophotometer system, formerly Hewlett Packard, now Agilent Technologies Finland Oy, Espoo) and chlorophyll a and b concentrations were calculated following Porra et al. (1989) (Table 1 and Appendix S2, Fig. S7).

Leaves for analysis of phenolic composition were air dried for 2 weeks following harvest. Three leaf disks are taken from seven seedlings from each treatment avoiding the major veins and these were ground together. Samples were extracted in high-performance liquid chromatography (HPLC)-grade methanol and analyzed following Keinänen and Julkunen-Tiitto (1996) and chromatographically separated using a Thermo Hypersil 


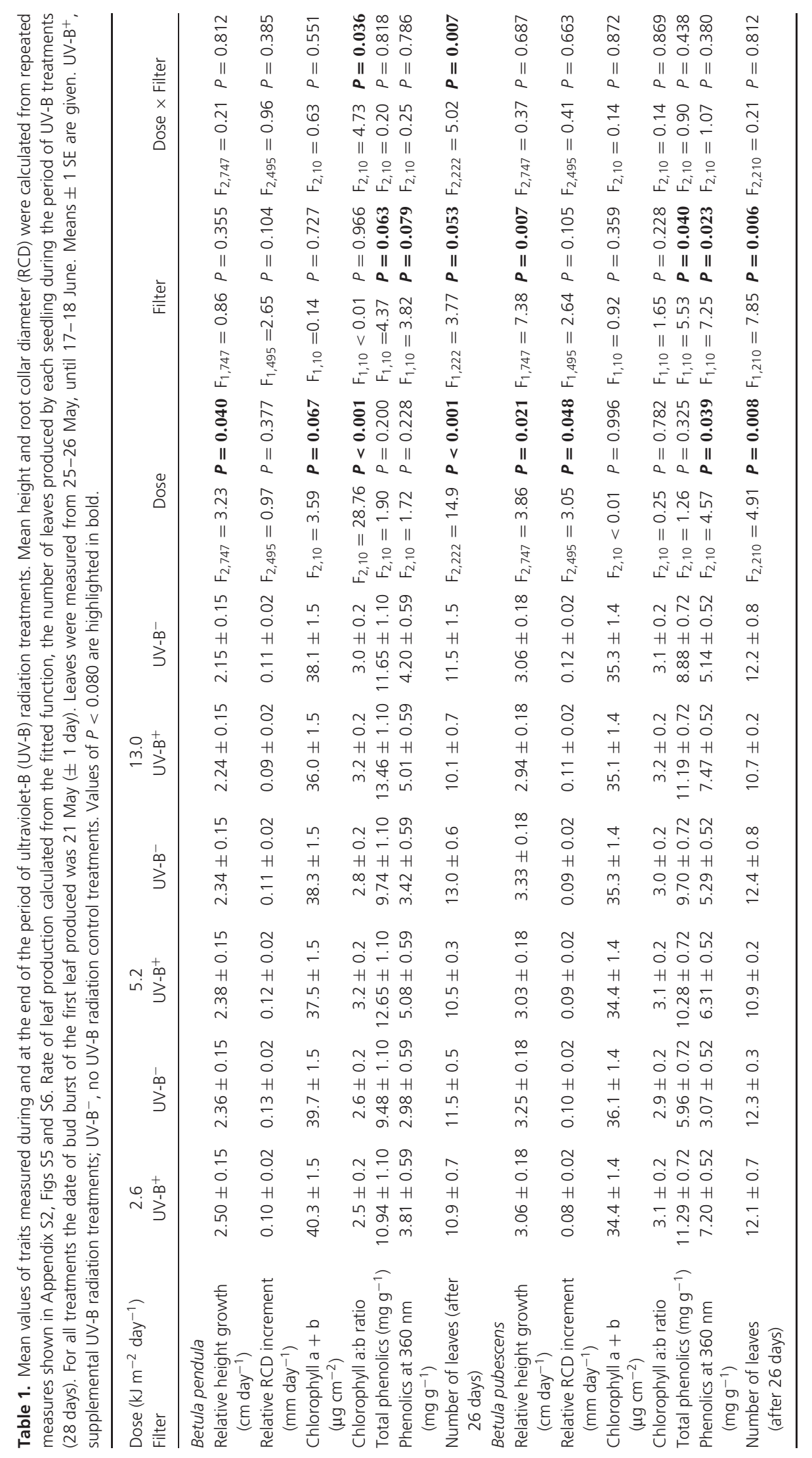

Physiol. Plant. 2012 
ODS column $(3 \mu \mathrm{m}, 4.6 \mathrm{~mm} \times 60 \mathrm{~mm}$, Thermo Electron Corporation, Bellefonte, PA) part of an Agilent HPLC system (1100 series) (Appendix S2, Figs S8 and S9).

\section{Data analysis - use of a two-segment smooth function}

Leaf growth data were analyzed by fitting a simple smooth function, which in the limit is a two-segment linear spline, with an initial sloping segment and a final horizontal segment (see Appendix S1 for details). To obtain biologically meaningful parameters for modeling leaf expansion data, we adapted the equation used by Prunty (1983 - Eqn 3.1 p 859). This function has previously been used to describe the time course of stomatal conductance during drought (Aphalo and Sánchez 1986) and for modeling leaf expansion (Aphalo et al. 2006) in both cases yielding biologically interpretable parameters. It is given by:

$$
W=A\left[1+d / A(t+b)-\left(d / A(t+b)^{c}+1\right)^{1 / c}\right]
$$

Using the notation for the parameters $\mathrm{t}$ is time in days and $W$ is the natural logarithm of leaf area in $\mathrm{mm}^{2}(\ln \mathrm{L})$.

$A$, natural $\log$ of $L_{\max }$, where $L_{\max }$ is the maximum leaf area $\left(\mathrm{mm}^{2}\right)$. It determines the upper asymptote $(\mathrm{t} \rightarrow \infty)$ (Appendix S1, Fig. S3).

$\mathrm{b}$, zero-time adjustment of the curve along the $\mathrm{x}$-axis, gives the different start dates of expansion when all measurements are referenced to the same initial calendar date (Appendix S1, Fig. S3).

c, the sharpness of curvature of the growth curve in its transition from exponential growth to cessation of growth at maximum area. At high values the transition is sharp and the exponential growth phase continues late into development (Appendix S1, Fig. S3).

$\mathrm{d} / \mathrm{A}$, the relative expansion rate of the exponential growth phase (Appendix S1, Fig. S3).

The parameters of the function were used to isolate different aspects of leaf growth such as (1) the relative rate of exponential leaf expansion $(\mathrm{d} / \mathrm{A}),(2)$ its duration and (3) the curvature, given by $\mathrm{c}$, indicating whether leaf expansion slows gradually or abruptly when close to its final size. Because the fit of the function to leaf expansion data is asymptotic at its upper limit, with variable sharpness of the transition, we were able to attribute a maximum size to leaves. It also allowed us to differentiate between delayed leaf expansion (because of $U V-B^{+}$) and any reduction in the final size of fully expanded leaves (Appendix S1, Fig. S2).

\section{Data analysis - statistical analysis}

The function (Eqn 1) was fitted to measurements on seven seedlings per block from each treatment combination by iteration from estimates of initial values chosen by visual inspection of the data (seven individuals $\times$ three leaves $x$ two species $x$ three blocks $x$ two UV-B/control $x$ three UV-B doses $=756$ fits: library nlme, nls procedure, $R$, version 2.8.1., $R$ Development Core Team 2008).

The experiment was laid out in a factorial design, and to account for any gradients in temperature, light and humidity the greenhouse was divided into three blocks with one replicate (seven individuals) of each treatment combination within each block. A mixed-model ANOVA was performed to determine treatment differences in the parameters of the fitted function. Filter $\left(U V-B^{+}\right.$treatment or $\mathrm{UV}^{-\mathrm{B}^{-}}$radiation control) and Dose (amount of UV-B radiation dependent on the irradiation time) and their interaction were fixed effect factors, and Block was a random effect factor. For those measurements taken from multiple leaves (leaves 1, 3 and 5) or on multiple occasions a three-way factorial ANOVA was performed to assess the overall responses, in addition to analysis of the effects on individual leaves or at single time points. The correct variance structure to use in the ANOVA model was determined by comparison of Akaike's Information Criterion and the log-likelihood ratio. Normality and homogeneity of variance of the residuals was visually assessed and data were natural log transformed when necessary. All statistical analyses were performed in $\mathrm{R}$ version 2.8.2 (R Development Core Team 2008).

\section{Results}

\section{Successfully fitting leaf growth}

By applying functional plant growth analysis, changes in leaf expansion rate, and the phase when these changes occurred, were distinguished with precision which would have been difficult to otherwise achieve. The refined Prunty (1983) function, that is piecewise linear in the limit, provided a better and more interpretable fit to our leaf expansion data than the other functions tested (Appendix S1, Fig. S1). There were very few problems fitting growth data from leaves 1 and 3 , leaves which were measured nine times each over a period of 24 days. Leaf 5 was measured on eight occasions over a period of 21 days and in $20-25 \%$ of cases could not be adequately modeled because it had not reached full size in this time. This difficulty could have been overcome by making a measurement of leaf size 1 week after the expected end of leaf growth to reduce uncertainty over the upper asymptote. The treatment effects on leaf 5 were statistically weaker than for the other two leaves, because fewer fits were obtained, consequently the UV effect on growth of just leaves 1 and 3 are given in Figs $1-4$. 


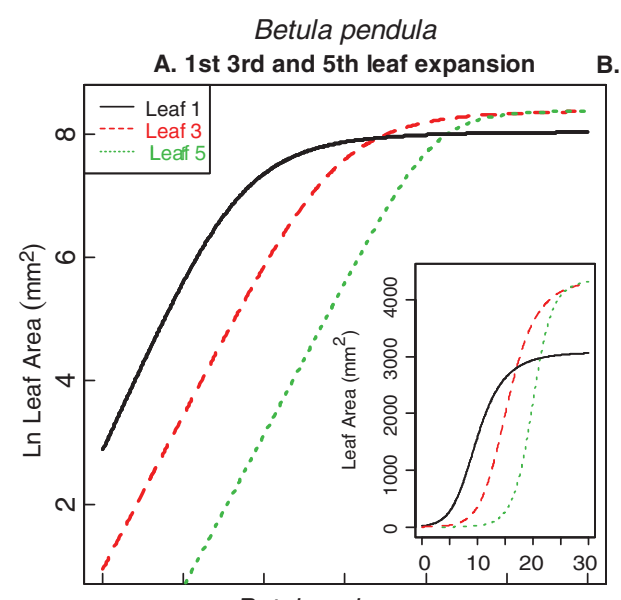

Betula pendula
B. Leaf expansion of UV-B treatments vs UV-A controls

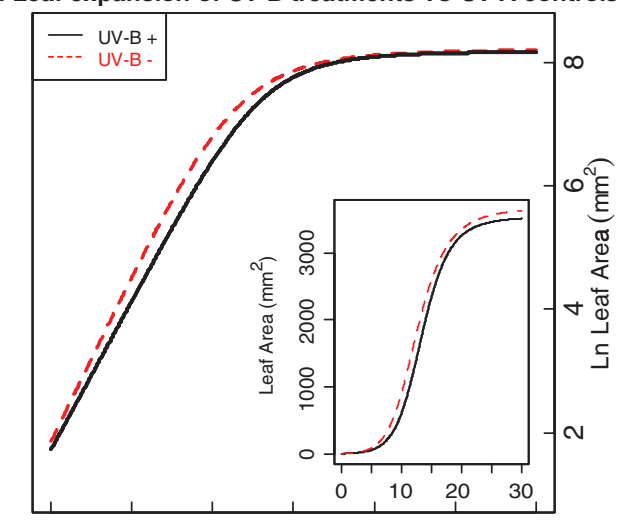

Betula pubescens

Betula pubescens

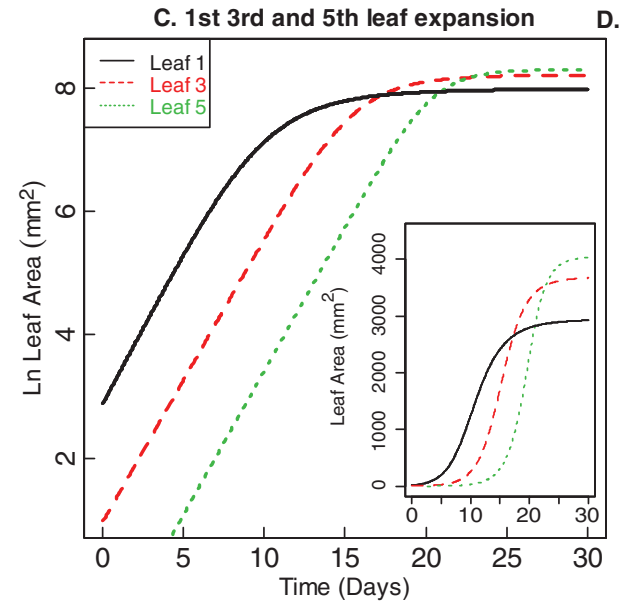

D. Leaf expansion of UV-B treatments vs UV-A controls

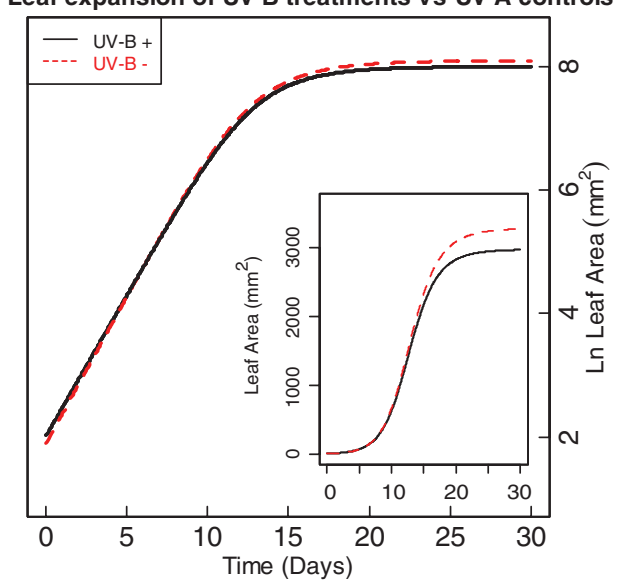

Fig. 1. Plotted functions illustrating the sequential production of leaves and effects of ultraviolet-B (UV-B) radiation treatment on leaf growth. The overall means of each parameter from fits to individual leaves are used to model growth under each treatment combination: Betula pendula (A) timing of leaf production for leaves $1(n=121), 3(n=116)$ and $5(n=96)$; (B) supplemental UV-B radiation treatments (UV-B $\left.B^{+}\right)$vs no UV-B radiation control treatments with filters blocking all the UV-B radiation but transmitting the small amount of UV-A radiation from the lamps (UV- $\left.B^{-}\right)$for leaves 1 and $3(n=222)$ : Betula pubescens (C) timing of leaf production for leaves $1(n=119), 3(n=109)$ and $5(n=100)$, (D) UV-B $B^{+}$treatment vs no UV-B radiation control $\left(U V-B^{-}\right)$for leaves 1 and $3(n=214)$. The variability around the fit is given for each parameter of the function in Figs 3 and 4 .

\section{Effects of UV-B radiation on leaf growth as illustrated by the fitted function}

In both species, leaf 1 (the 1 st distal leaf) was relatively small, and there was gradual increase in final size (A) with successive leaves produced (Fig. 1A, C). The rate of leaf expansion in both species was slower in leaves 3 and 5 compared with leaf 1 (Figs $1 \mathrm{~A}, \mathrm{C}, 3 \mathrm{C}$ and $4 \mathrm{C}$ ).

Our assessment revealed contrasting effects of UV-B radiation on different aspects of leaf expansion in the two species. There was no significant effect of UV-B ${ }^{+}$treatment (filter) on the final size of $B$. pendula leaves (Figs 1B and 3A; Table 2) but there was a reduction in the final size of B. pubescens leaves (Figs 1D and 4A; Table 2). These trends are apparent from leaf expansion curves of the mean of all measurements for each treatment
(Figs 1 and 2), but by using the parameters of the fitted piece-wise linear function we were able to separately test for differences between the effect of UV-B radiation on expansion rate and on final leaf size (Figs 3 and 4; Table 2). The parameter giving leaf final size (A) was $8.3 \%$ smaller overall for $B$. pubescens under the UV- $\mathrm{B}^{+}$ treatments than the UV- $\mathrm{B}^{-}$controls: this effect was similar in leaves 1 and 3, but more apparent at the higher UV-B doses (Fig. 3). In contrast, for B. pendula final size (A) was not significantly affected by UV-B ${ }^{+}$treatment, although there was a significant effect of decreasing size with increasing dose, the lack of a dose $\times$ filter interaction signified that this effect was also apparent under the no UV-B controls (Fig. 3; Table 2).

However, for $B$. pendula the rate of leaf expansion was slowed by the UV-B ${ }^{+}$treatments (Figs 3 and 4; 

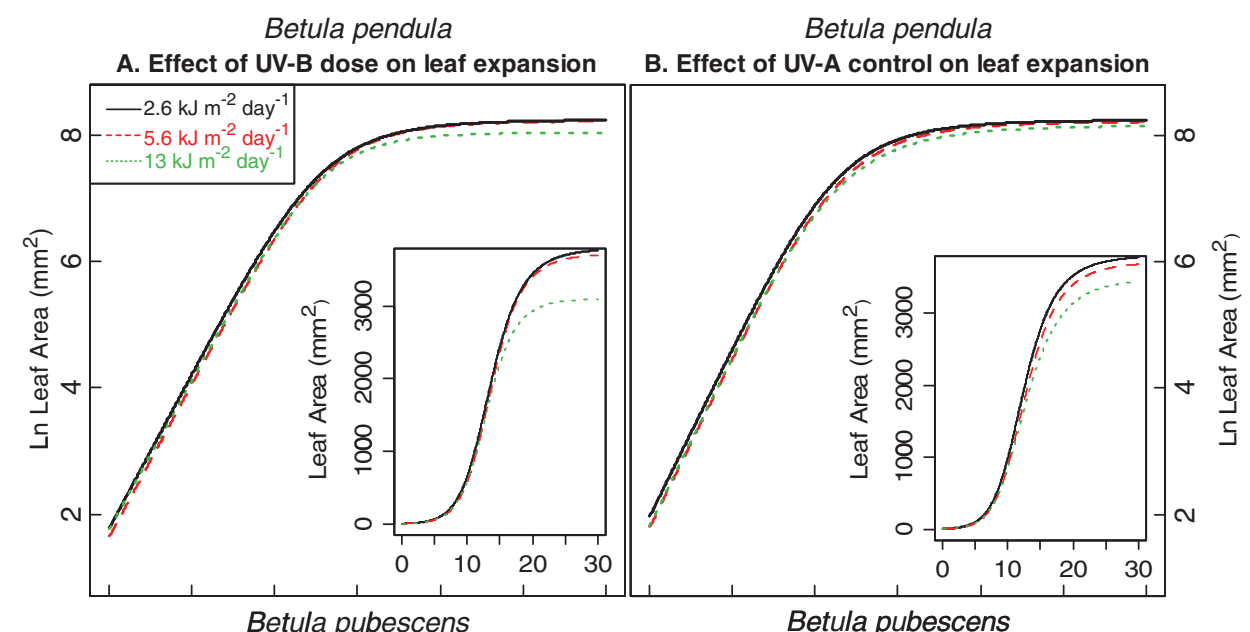

C. Effect of UV-B dose on leaf expansion
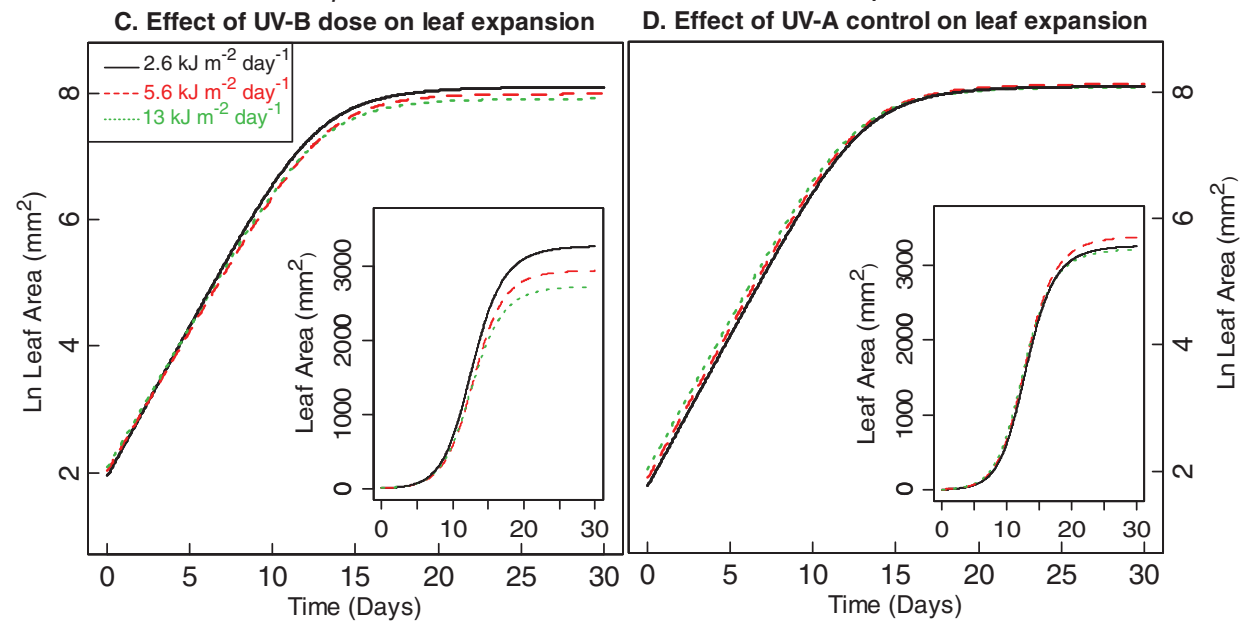

Fig. 2. Plotted functions illustrating the effects of ultraviolet-B (UV-B) radiation of tmr on leaf growth. The overall means of each parameter from fits to individual leaves (leaves 1 and 3 ) are used to model growth under each treatment combination: Betula pendula (A) three doses from the supplemental UV-B radiation treatments $\left(U V-B^{+}\right)$are compared $(n=222)$; $(B)$ three UV-A controls receiving no UV-B radiation (UV- $\left.B^{-}\right)$under the three UV-B doses are compared $(n=222)$ : Betula pubescens $(C)$ three doses from the UV- $B^{+}$treatment are compared $(n=214)$; $(D)$ three UV-A controls receiving no UV-B radiation $\left(U V-B^{-}\right)$under the three UV-B doses are compared $(n=214)$ The variability around the fit is given for each parameter of the function in Figs 3 and 4.

Table 2). The maximum growth rate $(\mathrm{d} / \mathrm{A})$, the curvature (c) and the time period between 10 and $90 \%$ expansion $(\delta \mathrm{t})$ all differed in response to $\mathrm{UV}-\mathrm{B}^{+}$treatment in $B$. pendula (Fig. 3; Table 2). The maximum leaf growth rate of $B$. pendula $(\mathrm{d} / \mathrm{A})$ was slowed by UV-B radiation compared to the no UV-B controls, but was not particularly affected by increasing the UV-B dose. The curvature (c) was higher, representing a sharper transition from exponential growth to maximum size, for the leaves receiving UV-B radiation $(P<0.001)$ and this effect did increase with UV-B dose (Fig. 2; Table 2 UV-B $\times$ Dose, $P=0.031$ ). The slower growth rate, $\mathrm{d} / \mathrm{A}$, in leaves under the UV-B ${ }^{+}$treatments was compensated by a prolonged period of exponential growth before its decline compared to those seedlings under the no UV-B controls, resulting in a similar final leaf size between $U V-B^{+}$and UV-B- treatments (Fig. 1; Table 2).

Despite a smaller final leaf size, in B. pubescens the effects of the UV-B ${ }^{+}$treatments on the leaf expansion parameters were less pronounced than for $B$. pendula and only for the maximum leaf growth rate was the effect of UV-B ${ }^{+}$treatment statistically significant (Fig. 4; Table 2). Although the time period between 10 and $90 \%$ expansion $(\delta \mathrm{t})$ was affected by the irradiation time as was the curvature (Fig. 4; Table 2), there were no differences in these parameters between the treatment and control filters even at the highest UV-B ${ }^{+}$dose (Fig. 4).

By using $x$-shift (b) to estimate the date of leaf emergence it was also possible to illustrate a decline in the rate of leaf production, given as the number of 

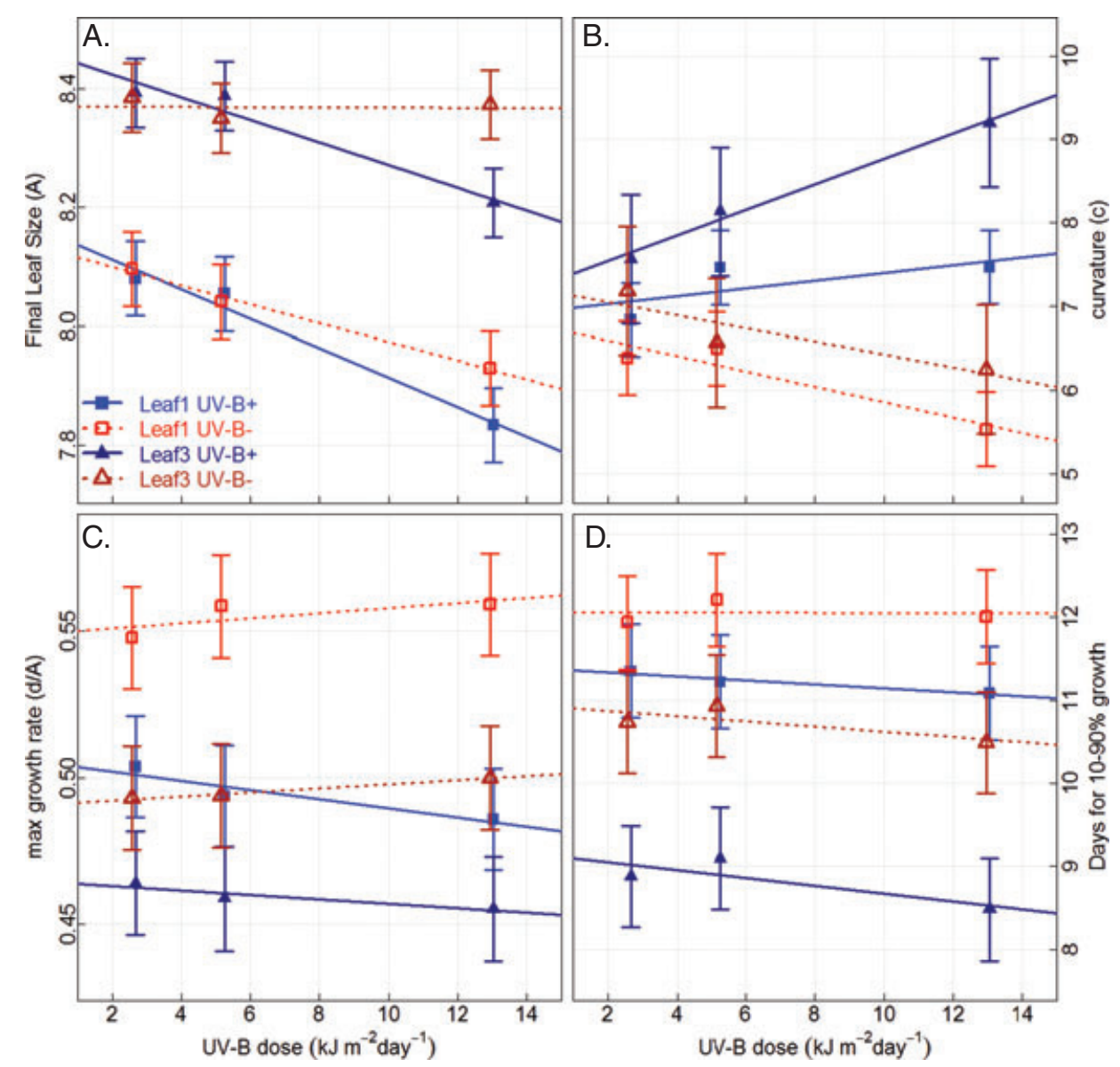

Fig. 3. Mean and SE of parameters from fitted functions for Betula pendula. The first and third leaves under each treatment combination are plotted.

leaves after 26 days, as a result of the UV-B ${ }^{+}$treatment (Table 1). In B. pubescens, there was a statistically significant effect of UV-B filter on leaf production, although this effect was not apparent at the lowest UV-B dose (Table 2). This effect of UV-B filter on leaf production was marginally non-significant in B. pendula (Table 2), and for both species there was a difference due to UV-B dose (Table 2).

The length:width ratio of leaves gradually decreased in both species as leaves grew (Fig. 5 and Appendix S2, Fig. S4). Leaf 1 was more elongated than leaves 3 and 5, and $B$. pendula leaves were more elongated than those of $B$. pubescens. The length:width ratio was only affected in B. pendula leaves during their first week of expansion following bud burst, with more elongated narrower leaves under the $U V-B^{+}$treatment, but this effect was transient and relatively small (Fig. 5). In B. pubescens, UV-B filter did not affect the leaf length:width ratio but this ratio did increase with $U V-B^{+}$dose; which could be interpreted as an affect of UV-A radiation (Fig. 5). There were no differences in teeth size nor visible chlorosis/necrosis of leaves between any of the treatments (data not shown).

\section{Effects of UV-B radiation on seedling growth, leaf pigments and phenolics}

Growth responses of both species to the UV-B ${ }^{+}$ treatments are detailed in the Appendix S2 and summarized in Table 1. Increments in RCD were small over the course of the experiment, and generally did not respond to the UV-B ${ }^{+}$treatments in either species. A tendency toward divergence under the two filter types suggested that with a longer period of exposure $B$. pubescens RCD would be reduced by UV-B radiation (Appendix S2, Fig. S5). The relative growth rate in height was reduced by the UV-B ${ }^{+}$treatment in $B$. pubescens only, not $B$. pendula, but this reduction was relatively small $\left(6.2 \%\right.$ mean decrease because of $U \mathrm{UV}^{+} \mathrm{B}^{+}$over 28 days in $B$. pubescens).

Leaf chlorophyll content was largely unaffected by UV-B radiation, although there was some indication of 


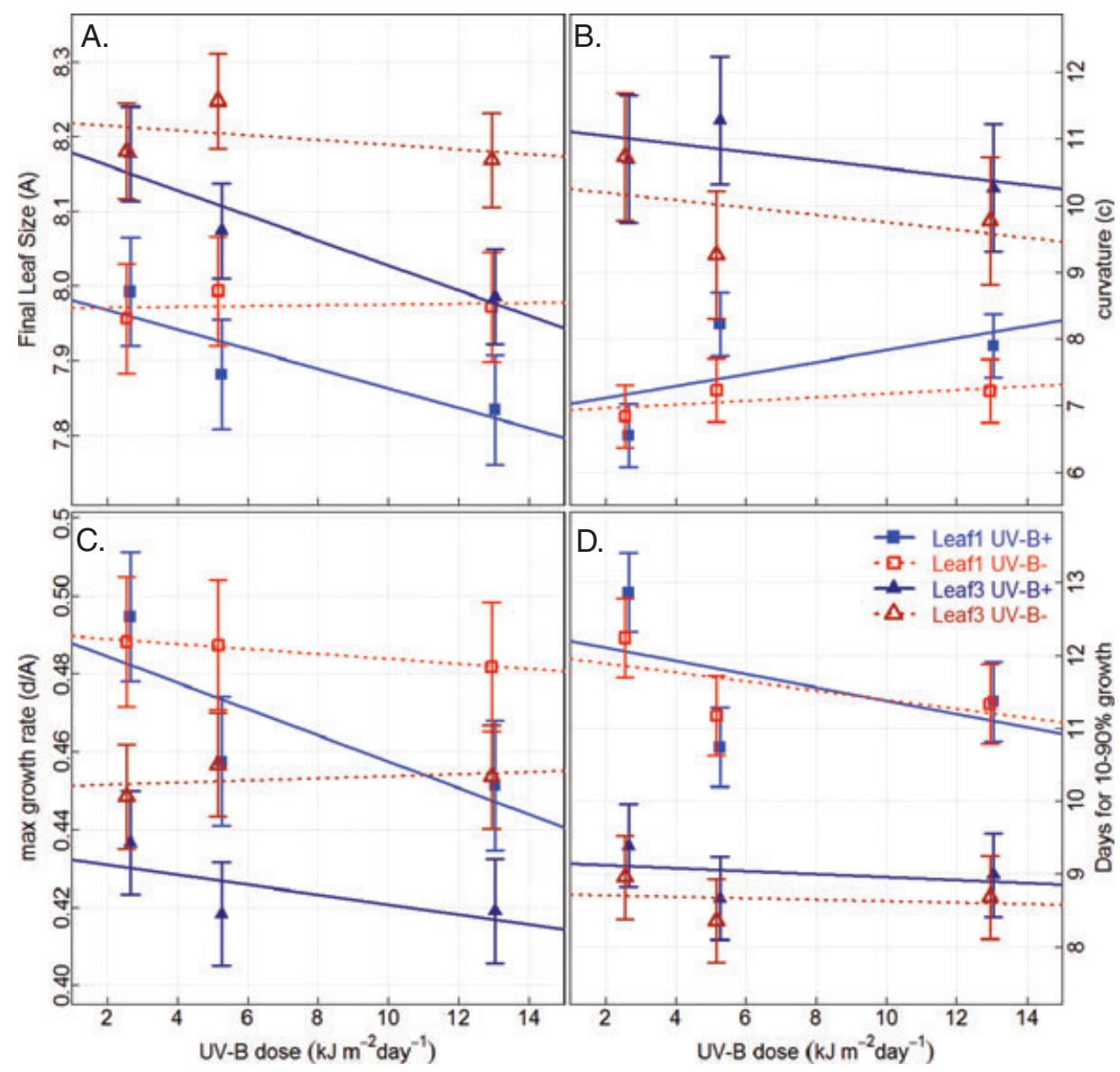

Fig. 4. Mean and SE of parameters from fitted functions for Betula pubescens. The first and third leaves under each treatment combination are plotted.

a decrease in chlorophyll content with increasing UV dose in B. pendula only (Table 1 ). Total leaf phenolic content increased in response to UV-B ${ }^{+}$in both species, but more in B. pubescens than B. pendula (Table 1). In both species, HPLC revealed quercetin derivatives to be more common than myricetin or kaempferol flavonoids. Quercetin 3-galactoside was the only compound to increase in concentration because of UV-B radiation in both species, but a suite of other flavonoids also increased as a result of UV-B radiation in B. pubescens (Appendix S2).

\section{Discussion}

\section{Do seedlings of $B$. pendula and $B$. pubescens respond similarly to UV-B radiation?}

The response of the two species to UV-B radiation differed, in that the final leaf size of $B$. pubescens was reduced by UV-B radiation, whereas leaf growth rate was slowed in $B$. pendula but with little effect of final leaf size. Our result that final leaf area of $B$. pendula was unaffected by UV-B radiation agrees with other published data for this species (Lavola et al. 1997, Kostina et al. 2001), however, previous studies of $B$. pendula have not examined leaf growth in such detail as to identify the effects during expansion which we were able to isolate through functional growth analysis.

There is some evidence for reduced leaf size caused by UV-B radiation in other broad-leaved tree species: Nothofagus solandri produced smaller firstflush leaves under ambient UV-B radiation compared with reduced UV-B radiation in New Zealand, but this was counterbalanced by greater leaf production in the second flush (Hunt and McNeil 1999).

\section{Which phases of leaf growth respond to UV-B radiation?}

The difference in the effect of UV-B radiation on the two species suggests that in $B$. pendula a regulatory response diverts resources during leaf expansion but 


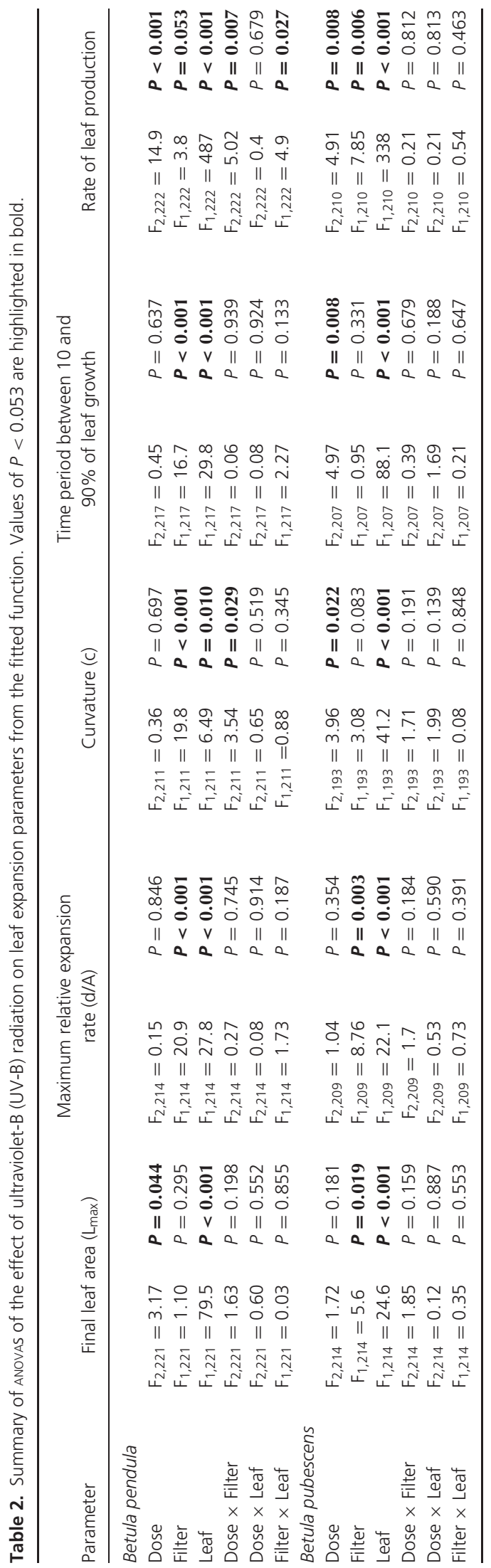

that the leaf compensates by maintaining its maximum growth rate for longer followed by a sharper transition before the cessation of growth (Figs 1 and 3). The fact that these changes during leaf expansion of $B$. pendula were unaffected by the size of $U V-B^{+}$dose supports the idea that they are regulatory responses activated at low doses rather than a result of UV-B radiation damage. For $B$. pubescens, the situation differed somewhat from $B$. pendula, because there was no UV-B effect on the transition from maximum growth rate to final size (c: Fig. 4) but more particularly that leaf final sizes were decreased as UV-B ${ }^{+}$dose increased (Fig. 4).

Does this difference between the species reflect different mechanisms of response to UV-B radiation or is it simply because of the magnitude of response? Most cell division in birch is expected to have already occurred before leaves emerge from their bud scales, but premature emergence can lead to a smaller final size and the previous years' conditions influence the primordia of the first leaves produced (Cutter 1971). Reduced leaf size in $A$. thaliana under UV-B radiation treatments was attributed to reduced cell expansion because of a temporary reallocation of resources and was associated with changes in the leaf length:width ratio (Hectors et al. 2010). Here the leaf length:width ratio in B. pendula was transiently increased by UV-B radiation early in growth, whereas in $B$. pubescens it was not affected. Although the difference in leaf length:width ratio in $B$. pendula caused by UV-B radiation was in the opposite direction to that reported by Hectors et al. (2010), it nevertheless may reflect that cell expansion was affected by UV-B radiation and that this effect was compensated for before the completion of leaf growth as it coincides with the transient effects on leaf expansion rate that we report. Compensatory responses during different phases of leaf growth have been reported elsewhere (Barkan et al. 2006, Wargent et al. 2009a). In B. pubescens, the absence of a change in leaf length:width ratio as a result of UV-B radiation and lack of difference in growth rate during expansion, may suggest that a difference in cell number rather than cell expansion could be responsible for the decreased final size in this species. Predominant effects of UV-B radiation on cell division over expansion have been reported for Rumex patientia (Dickson and Caldwell 1978) and Pisum sativum (Gonzalez et al. 1998). Further molecular and physiological studies would be needed to confirm the mechanisms behind the photomorphogenic effects of UV-B radiation (Jansen 2002).

There was an effect of dose, but no effect of, or interaction with, filter, for duration of leaf expansion in $B$. pubescens. This dose effect is seemingly because of the small amount of UV-A produced by the 


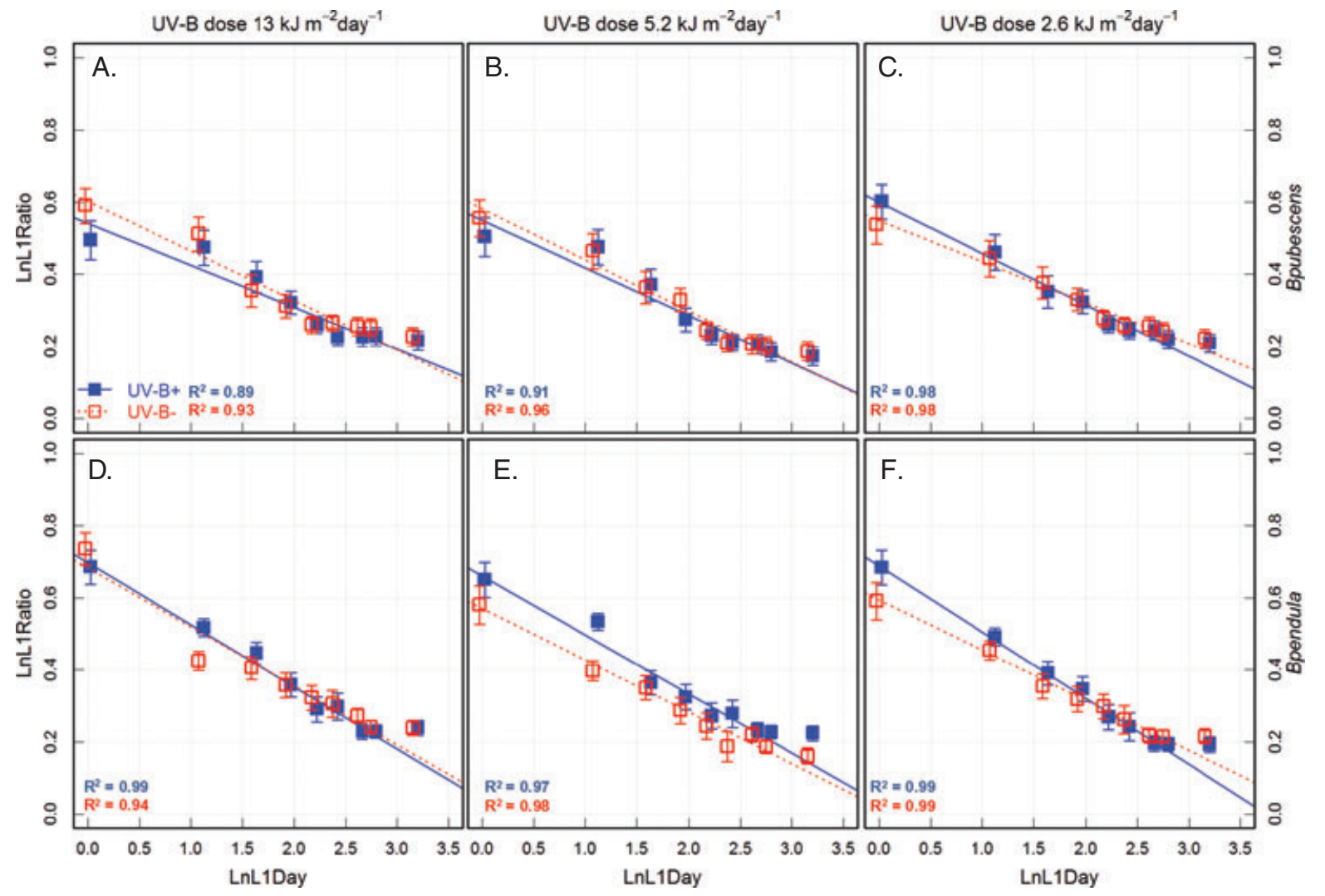

Fig. 5. Change in leaf length:width ratio of leaves over the course of the ultraviolet (UV) treatments: The first leaf produced is plotted for each pair of treatments ( $n=222$ Betula pendula, $n=214$ Betula pubescens). The third and fifth leaves produced followed similar patterns (Appendix S2, Fig. S4).

lamps and transmitted through both filter types (Fig. 4; Table 2), although other effectors like slight differences in temperature produced by energized lamps cannot be completely ruled out. Similar UV-A effects have been reported elsewhere (Middleton and Teramura 1993, Newsham et al. 1996, McLeod 1997), and it has been suggested that UV-A has a distinct effect on cell proliferation to that of UV-B radiation during leaf expansion (Wargent et al. 2009b). This disparity could be a result of the different photoreceptors that mediate responses to UV-A and UV-B radiation, but to explain the significance of these differences for plant function will require greater understanding of the interactions between those pathways that mediate the responses within these wavebands.

\section{What are the implications for the wider UV-B radiation effects on birch species?}

The effects of UV-B radiation on leaf traits are generally more apparent as differences in leaf growth and morphology than effects on photosynthesis, as was the case in this study where no differences in leaf chlorophyll content occurred but species-specific reductions in leaf area were recorded. Often it takes several years for effects of UV-B radiation on leaf traits to be expressed as reductions in height growth (Robson et al. 2003, Tegelberg et al. 2004, Caldwell et al. 2007), but in this study measurements of height growth on six occasions over 1 month were sufficient to reveal differences between UV-B radiation treatments in B. pubescens. Although these effects of UV-B radiation were small, they may accumulate over the long life span of $B$. pubescens if the differences in the UV-B radiation environment were maintained. Just as this difference in growth scales up from leaf to plant height in $B$. pubescens, the height growth and RCD of $B$. pendula were not affected by UV-B radiation treatments and likewise neither was final leaf size.

Most previous studies have also failed to find any effects of UV-B radiation on $B$. pendula growth: neither under UV-B-supplementation in a greenhouse (de la Rosa et al. 2003), nor in UV-B-reduction field experiments (Keski-Saari et al. 2005, Kotilainen et al. 2009, Morales etal. 2010), and only after several years of treatments in a UV-B-supplementation field 
study was a reduction in growth evident (Kostina et al. 2001, Tegelberg et al. 2004). In short term experiments, extremely high UV-B radiation doses $\left(22.5 \mathrm{~kJ} \mathrm{~m}^{-2}\right.$ day $^{-1}$ ) have been required to produce impairment of B. pendula performance and visible symptoms of leaf damage (Wulff et al. 1999). The relative growth rate of $B$. pubescens ssp. czerepanovii (also know as ssp. tortuosa) in a greenhouse study similar to this one was reduced by enhanced UV-B radiation treatments but the effect was variable among different families (Weih et al. 1998).

B. pendula leaves contain more phenolic compounds and have a different phenolic composition from B. pubescens (Ossipov et al. 1996). Both species rely on both physiological and morphological adaptations to defend against UV-B radiation, such as leaf trichomes producing flavonoid aglycones on the epidermal surface (Kostina et al. 2001, Valkama et al. 2004). Increased production of phenolic glycosides in the leaves of B. pendula has been widely reported under UV-B radiation (Julkunen-Tiitto et al. 2005, Kotilainen et al. 2009, Morales et al. 2010), and some specific flavonoids, such as myricetin 3-galacoside (Lavola et al. 1997) and quercetin glycosides (Keski-Saari et al. 2005) have been identified as particularly responsive to UV-B radiation. Likewise, in B. pubescens particular flavonoids respond to UV-B radiation (Kotilainen et al. 2008). The results of HPLC analysis on the leaves measured in this experiment reaffirmed these patterns of response to UV-B and to UV-A radiation (Appendix S2). The timing of this increased synthesis of flavonoids may influence leaf expansion if phenolics content is low and leaves are exposed to UV-B radiation during the early stages of their development (Teramura and Caldwell 1981), although such affects have not been identified in B. pubescens czerepanovii (Riipi et al. 2002). During the initial development of Betula leaves, bud scales should protect them from UV-B radiation, but there is evidence that once leaves are exposed, flavonoids are differentially synthesized during their development (Morales et al. 2010). This temporal separation in phenolics synthesis in $B$. pendula may suggest the differential expression of genes within the phenylpropanoid pathway in response to UV-B radiation during the period of leaf growth (Morales et al. 2010).

In this study, all limiting factors to growth apart from UV-B radiation were minimized in order to eliminate possible confounding effects. In other experiments where UV-B radiation treatments have been combined with nutrient limitation, imposed drought and elevated $\mathrm{CO}_{2}$ concentrations interactive effects have generally reduced the influence of UV-B radiation on $B$. pendula and B. pubescens (Lavola et al. 1997, de la Rosa et al.
2001, 2003). Hence, the subtle effects that we reveal may be partially ameliorated when examined in the more-complex natural environment.

\section{Conclusions}

The effects of the UV-B radiation treatments were small but by fitting a curve covering the whole period of leaf growth we were able to pinpoint the different UV-B effect between species. The leaf final size was reduced in $B$. pubescens, as opposed to $B$. pendula where a prolongation of the growth period at a slower rate due to UV-B radiation did not significantly affect leaf final size. This result agrees with recent studies suggesting that leaf growth can be transiently affected by UV-B radiation because of changes in leaf expansion (Wargent et al. 2009b, Hectors et al. 2010). Transient effects during leaf growth indicate that UV-B exposure early in the growing season may be more important than UV-B radiation later in the summer once leaves are fully developed. They also indicate that both species have very efficient regulatory mechanisms that enable leaves to acclimate to $U V-B$ radiation in the environment and are activated even at low UV-B doses. Whereas, even when UV$B$ doses are increased to approach the upper limit of those received in nature, the consequences for whole plant performance are often negligible, at least in short duration experiments. No persistent negative effects of UV-B radiation were evident for leaf size or growth of $B$. pendula at the end of the treatments, whereas reduced leaf size in $B$. pubescens scaled up to a reduced rate of height growth indicating that the effects of UV-B radiation were congruent between scales.

Acknowledgements - This work was funded by the Maj and Tor Nessling Foundation (grant to P. J. A.), the Finnish Academy of Science (decision no. 116775 to P. J. A.) and the Spanish Ministry of Education and Science (Juan de la Cierva Fellowship to T. M. R.). We thank John Grace for his valuable advice in the preparation of this research, Riitta Julkunen-Tiitto whose lab performed the HPLC work, and Tania de la Rosa, Anu Lavola and Sanja Sorjonen for their practical help in performing the experiment. Marcel Jansen, Luis Morales and Tarja Lehto gave helpful comments on the text.

\section{References}

Aphalo PJ, Sánchez RA (1986) Stomatal responses to light and drought stress in variegated leaves of Hedera helix. Plant Physiol 81: 768-773

Aphalo PJ, Tegelberg R, Julkunen-Tiitto R (1999) The modulated UV-B irradiation system at the University of Joensuu. Biotronics 28: 109-120 
Aphalo PJ, Lehto M, Repo T, Rummukainen A, Mannerkoski H, Finér L (2006) Responses of silver birch saplings to low soil temperature. Silva Fenn 40: 429-442

Ballaré $\mathrm{CL}$, Caldwell MM, Flint SD, Robinson SA, Bornman JF (2010) Effect of solar ultraviolet radiation on terrestrial ecosystems patterns mechanisms and interactions with climate change (Chapter 3) In: Environmental Effects of Ozone Depletion and its Interactions with Climate Change: 2010 assessment. United Nations Environment Programme (UNEP), Nairobi, Kenya, pp 83-110

Barkan L, Evans MA, Edwards GE (2006) Increasing UV-B induces biphasic leaf cell expansion in Phaseolus vulgaris, suggesting multiple mechanisms for controlling plant growth. Photochem Photobiol 82: 1612-1620

Brown BA, Jenkins GI (2008) UV-B signalling pathways with different fluence-rate response profiles are distinguished in mature Arabidopsis leaf tissue by requirement for UVR8. HY5: and HYH Plant Physiol 146: 576-588

Caldwell MM (1971) Solar UV irradiation and the growth and development of higher plants. In: Giese AC (ed) Photophysiology. Academic Press, New York, pp 131-177

Caldwell MM, Bornman JF, Ballaré CL, Flint SD, Kulandaivelu G (2007) Terrestrial ecosystems increased solar ultraviolet radiation and interactions with other climate change factors. Photochem Photobiol Sci 6: 252-266

Cutter EG (1971) Plant anatomy: experiment and interpretation. Edward Arnold, London, pp 343

de la Rosa TM, Julkunen-Tiitto R, Lehto T, Aphalo PJ (2001) Secondary metabolites and nutrient concentrations in silver birch seedlings under five levels of daily UV-B exposure and two relative nutrient addition rates. New Phytol 150: 121-131

de la Rosa TM, Aphalo PJ, Lehto T (2003) Effects of ultraviolet- $B$ radiation on growth mycorrhizas and mineral nutrition of silver birch (Betula pendula Roth) seedlings grown in low-nutrient conditions. Global Change Biol 9: 65-73

Dickson JG, Caldwell MM (1978) Leaf development of Rumex-Patientia I (Polygonaceae) exposed to UV-B irradiation (280-320 nm). Am J Bot 65: 857-863

Favory JJ, Stec A, Gruber H, Rizzini L, Oravecz A, Funk M, Albert A, Cloix C, Jenkins GI, Oakeley EJ, Seidlitz HK, Nagy F, Ulm R (2009) Interaction of COP1 and UVR8 regulates $U V$-B induced photomorphogenesis and stress acclimation in Arabidopsis. EMBO J 28: 591-601

Flint SD, Caldwell MM (2003) A biological spectral weighting function for ozone depletion research with higher plants. Physiol Plant 117: 137-144

Gonzalez R, Mepsted R, Wellburn AR, Paul ND (1998) Non-photosynthetic mechanisms of growth reduction in pea (Pisum sativum L.) exposed to UV-B radiation. Plant Cell Environ 21: 23-32

Granier C, Tardieu F (2009) Multi-scale phenotyping of leaf expansion in response to environmental changes: the whole is more than the sum of parts. Plant Cell Environ 32: 1175-1184

Green AES, Sawada T, Shettle EP (1974) The middle ultraviolet reaching the ground. Photochem Photobiol 19: $251-259$

Hectors K, Prinsen E, De Coen W, Jansen MAK, Guisez Y (2007) Arabidopsis thaliana plants acclimated to low dose rates of ultraviolet $\mathrm{B}$ radiation show specific changes in morphology and gene expression in the absence of stress symptoms. New Phytol 175: 255-270

Hectors K, Jacques E, Prinsen E, Guisez Y, Verbelen J-P, Jansen MAK, Vissenberg K (2010) UV radiation reduces epidermal cell expansion in leaves of Arabidopsis thaliana. J Exp Bot 61: 4339-4349

Hunt JE, McNeil DL (1999) The influence of present-day levels of ultraviolet-B radiation on seedlings of two Southern Hemisphere temperature tree species. Plant Ecol 143: 39-50

Hunt R (2003) Plant growth analysis: individual plants. In: Thomas B, Murphy DJ, Murray D (eds) Encyclopedia of applied plant sciences. Academic Press, New York and London, pp 579-588

Jansen MAK (2002) Ultraviolet-B radiation effects on plants: induction of morphogenic responses. Physiol Plant 116: 423-429

Jenkins GI (2009) Signal transduction in responses to UV-B radiation. Annu Rev Plant Biol 60: 407-431

Julkunen-Tiitto R, Häggman H, Aphalo PJ, Lavola A, Tegelberg R, Veteli T (2005) Growth and defense in deciduous trees and shrubs under UV-B. Environ Pollut 137: 404-414

Keski-Saari S, Pusenius J, Julkunen-Tiitto R (2005) Phenolic compounds in seedlings of Betula pubescens and $B$. pendula are affected by enhanced UVB radiation and different nitrogen regimens during early ontogeny. Global Change Biol 11: 1180-1194

Keinänen M, Julkunen-Tiitto R (1996) Effect of sample preparation method on birch (Betula pendula Roth) leaf phenolics. J Agric Food Chem 44: 2724-2727

Kostina E, Wulff A, Julkunen-Tiitto R (2001) Growth, structure, stomatal responses and secondary metabolites of birch seedlings (Betula pendula) under elevated UV-B radiation in the field. Trees 15: 483-491

Kotilainen T, Tegelberg R, Julkunen-Tiitto R, Lindfors A, Aphalo PJ (2008) Metabolite specific effects of solar UV-A and UV-B on alder and birch leaf phenolics. Global Change Biol 14: 1-11

Kotilainen $\mathrm{T}$, Venäläinen $\mathrm{T}$, Tegelberg R, Lindfors A, Julkunen-Tiitto R, Sutinen S, O'Hara RB, Aphalo PJ (2009) Assessment of UV biological spectral weighting functions for phenolic metabolites and growth responses 
in silver birch seedlings. Photochem Photobiol 85: 1364-1355

Kozlowski TT, Clausen JJ (1966) Shoot growth characteristics of heterophyllous woody plants. Can J Bot 44: 827-843

Kvêt J, Marshall JK (1971) Assessment of leaf area and other assimilating plant surfaces. In: Sestak Z, Catsky J, Jarvis PG (eds) Plant Photosynthetic Production: Manual of Methods. Dr W Junk NV, The Hague, pp 517-555

Lavola A (1997) Accumulation of flavonoids and related compounds in birch induced by UV-B irradiance. Tree Physiol 18: 53-58

Lavola A, Julkunen-Tiitto R, Aphalo PJ, de la Rosa TM, Lehto T (1997) The effect of UV-B radiation on UV-absorbing secondary metabolites in birch seedlings grown under simulated forest soil conditions. New Phytol 137: 617-621

McLeod AR (1997) Outdoor supplementation systems for studies of the effects of increased UV-B radiation. Plant Ecol 128: 78-92

Middleton EM, Teramura AH (1993) The role of flavonoid glycosides and carotenoids in protecting soybean from ultraviolet-B damage. Plant Physiol 103: 741-752

Morales LO, Tegelberg R, Brosché $M$, Keinänen $M$, Lindfors A, Aphalo PJ (2010) Effects of solar UV-A and UV-B radiation on gene expression and phenolics accumulation in Betula pendula leaves. Tree Physiol 30: 923-934

Newsham KK, McLeod AR, Greenslade PD, Emmett AA (1996) Appropriate controls in outdoor UV-B supplementation experiments. Global Change Biol 2: 319-324

Ossipov V, Nurmi K, Loponen J, Prokopiev N, Haukioja E, Pihlaja K (1996) High-performance liquid chromatographic separation and identification of phenolic compounds from leaves of Betula pubescens and Betula pendula. J Chromatography A 721: 59-68

Porra RJ, Thompson WA, Kriedmann PE (1989) Determination of accurate extinction coefficients and simultaneous equations for assaying chlorophyll $\mathrm{a}$ and $\mathrm{b}$ extracted with four different solvents: verification of the concentration of chlorophyll standards by atomic absorption spectroscopy. Biochemica et Biohysica Acta 975: 384-393

Prunty L (1983) Curve fitting with smooth functions that are piecewise-linear in the limit. Biometrics 39: 857-866

R Development Core Team (2009) R: A Language and Environment for Statistical Computing. R Foundation for Statistical Computing, Vienna

Robson TM, Pancotto VA, Flint SD, Ballaré CL, Sala OE, Scopel AL, Caldwell MM (2003) Six years of solar UV-B manipulations affect growth of Sphagnum and vascular plants in a Tierra del Fuego peatland. New Phytol 160: 379-389
Riipi M, Ossipov V, Lempa K, Haukioja E, Koricheva J, Ossipova S, Kalevi P (2002) Seasonal changes in birch leaf chemistry: are there trade-offs between leaf growth and accumulation of phenolics? Oecologia 130: 380-390

Tardieu F, Granier C, Muller B (1999) Modelling leaf expansion in a fluctuating environment: are changes in specific leaf area a consequence of change in expansion rate? New Phytol 143: 33-43

Tegelberg R, Julkunen-Tiitto R, Aphalo PJ (2004) Red: far-red light ratio and UV-B radiation: their effects on leaf phenolics and growth of silver birch seedlings. Plant Cell Environ 27: 1005-1013

Teramura AH, Caldwell MM (1981) Effects of ultraviolet-B irradiances on soybean IV leaf ontogeny as a factor in evaluating ultraviolet-B irradiance effects on net photosynthesis. Am J Bot 68: 934-941

Valkama E, Salminen JP, Koricheva J, Pihlaja K (2004) Change in leaf trichomes and epicuticular flavonoids during leaf development in three birch taxa. Ann Bot 94: 233-242

Wargent JJ, Gegas VC, Jenkins GI, Doonan JH, Paul ND (2009a) UVR8 in Arabidopsis thaliana regulates multiple aspects of cellular differentiation during leaf development in response to ultraviolet $\mathrm{B}$ radiation. New Phytol 183: 315-326

Wargent JJ, Moore JP, Ennos AR, Paul ND (2009b) Ultraviolet radiation as a limiting factor in leaf expansion and development. Photochem Photobiol 85: 279-286

Weih M, Johanson U, Gwynn-Jones D (1998) Growth and nitrogen utilization in seedlings of mountain birch (Betula pubescens ssp. tortuosa) as affected by ultraviolet radiation (UV-A and UV-B) under laboratory and outdoor conditions. Trees 12: 201-207

Wulff A, Anttonen S, Pellinen R, Savonen EM, Sutinen ML, Heller W, Sanderman HJ, Kangasjärvi J (1999) Birch (Betula pendula Roth.) responses to high UV-B radiation. Boreal Environ Res 4: 77-88

\section{Supporting Information}

Additional Supporting Information may be found in the online version of this article:

Table S1. Summary ANOVA of effects of UV-B treatments on leaf length:width ratio.

Table S2. Summary ANOVA of effects of UV-B treatments on individual leaf phenolic contents.

Fig. S1. Comparison of four fitted functions to expansion of a typical Betula pendula leaf.

Fig. S2. The piece-wise linear function fitted to the same data as Fig. S1. 
Fig. S3. Sensitivity of the piece-wise linear function to changes in the parameters.

Fig. S4. Change in leaf length:width ratio of the third leaf produced (L3) over the course of the UV treatments.

Fig. S5. Change in the leaf length:width ratio of the fifth leaf produced (L5) over the course of the UV treatments.

Fig. S6. Increment in root collar diameter (RCD in $\mathrm{mm}$ ) over the course of the UV treatments.

Fig. S7. Increment in height growth $(\mathrm{cm})$ over the course of the UV treatments.

Fig. S8. Relationship between individual leaf phenolic compounds in Betula pendula extracted and analysed by HPLC at the end of the period of UV treatments and dose.

Fig. S9. Relationship between individual leaf phenolic compounds in B. pubescens extracted and analysed by HLPC at the end of the period of UV treatments and dose.

Appendix S1. Plant growth analysis.

Appendix S2. Complementary data.

Please note: Wiley-Blackwell are not responsible for the content or functionality of any supporting materials supplied by the authors. Any queries (other than missing material) should be directed to the corresponding author for the article. 